\title{
Maxwell and Scalar Fields in Nonstandard Cosmology
}

\author{
Günter Scharf \\ Physics Institute, University of Zürich, Winterthurerstr. 190, CH-8057 Zürich, Switzerland \\ Email: scharf@physik.uzh.ch
}

\begin{abstract}
We study the scalar field equation and Maxwell equations in the nonstandard background previously investigated. We separate the angular dependence by expanding in spherical harmonics and solve the radial wave equations exactly by separating the variables $t$ and $r$. We obtain electric and magnetic multipole radiation as in vacuum without gravitation, but the frequencies get redshifted. The exact results are essential for understanding the Planckian character of CMB properly.
\end{abstract}

Keywords: cosmology

\section{Introduction}

In standard cosmology radiation is important at early times only. If dark matter does not exist the situation is quite different. The reason is that the energy density of radiation grows rapidly with the forth power of the temperature $\propto T^{4}$, while for non-relativistic matter it varies only linearly $\propto T$. To get some numbers we take the present baryon density of 0.04 times the critical density. This gives an energy density

$$
n_{B} m_{B}=3.31 \times 10^{-10} \frac{\mathrm{erg}}{\mathrm{cm}^{3}} .
$$

The same energy density produced by radiation requires a temperature

$$
T_{R}=\left(\frac{n_{B} m_{B}}{a_{B}}\right)^{1 / 4}
$$

where $a_{B}$ is the Stefan-Boltzmann radiation constant. For the value (1.1) we obtain $T_{R}=14.5^{\circ} \mathrm{K}$. This surprisingly small temperature shows that in cosmology without dark matter electromagnetic radiation is the most important player beside gravity.

If the energy density of matter and radiation is small compared to the critical density then a good starting point for cosmology is a vacuum solution of Einstein's equation. Assuming spherical symmetry this solution is the inner Schwarzschild solution in Lemaitre coordinates as a consequence of Birkhoff's theorem. Matter is then treated as anisotropic perturbation away from this nonstandard background. This was investigated in two papers [1], [2] with the following results. The anisotropic perturbations with spherical harmonic order $l>1$ come from pressure-less dust, but the isotropic perturbations $l=0$ could not be calculated. Of course one immediately presumes that the latter is due to CMB. To test this conjecture we must study Maxwell equations in the nonstandard background which is the purpose of this article.

The paper is organized as follows. As warming up we first consider a scalar field in the nonstandard background. Here the angular variables can be trivially separated by means of scalar spherical harmonics. In sect. 3 we turn to Maxwell equations which are transformed in a similar way as in Minkowski space. Then in sect. 4 and 5 the angular dependence is separated by using vector spherical harmonics and the radial wave equations are solved. In the last section the physics of the multipole radiation is discussed. We conclude that Planck's radiation law for CMB is a consequence of the constancy of the density of photon states under the cosmic expansion. Together with the redshift of frequencies or temperature, the phase velocity of light is changed by the factor $(1+z)$. 


\section{Real Scalar Field in Nonstandard Background}

We choose spherical comoving coordinates $t, r, \vartheta, \phi$. Then the line element of the nonstandard background reads [1]

$$
d s^{2}=d t^{2}-X(t)^{2} d r^{2}-R(t)^{2}\left(d \vartheta^{2}+\sin ^{2} \vartheta d \phi^{2}\right) .
$$

with

$$
\dot{R}(t)=X(t)
$$

The corresponding metric tensor is equal to

$$
g_{\mu \nu}=\operatorname{diag}\left(1,-X^{2}(t),-R^{2}(t),-R^{2}(t) \sin ^{2} \vartheta\right) .
$$

We first consider a real massive scalar field in this background which satisfies the Laplace-Beltrami equation

$$
\square_{g} \varphi=\frac{1}{\sqrt{g}} \frac{\partial}{\partial x^{\alpha}}\left(\sqrt{g} g^{\alpha \beta} \frac{\partial \varphi}{\partial x^{\beta}}\right)=-m^{2} \varphi .
$$

This can be considered as a model of the matter in the otherwise empty universe. Since

$$
\sqrt{g}=X R^{2} \sin \vartheta
$$

the field equation becomes

$$
\begin{gathered}
\frac{1}{X R^{2} \sin \vartheta}\left[\partial_{0}\left(X R^{2} \sin \vartheta \partial_{0} \varphi\right)-\partial_{1}\left(\frac{R^{2} \sin \vartheta}{X} \partial_{1} \varphi\right)-\right. \\
\left.-\partial_{2}\left(X \sin \vartheta \partial_{2} \varphi\right)-\partial_{3}\left(\frac{X}{\sin \vartheta} \partial_{3} \varphi\right)\right]= \\
=\frac{1}{X R^{2}} \partial_{t}\left(X R^{2} \partial_{t} \varphi\right)-\frac{1}{X^{2}} \partial_{r}^{2} \varphi- \\
-\frac{1}{R^{2} \sin \vartheta} \partial_{\vartheta}\left(\sin \vartheta \partial_{\vartheta} \varphi\right)-\frac{1}{R^{2} \sin ^{2} \vartheta} \partial_{\phi}^{2} \varphi=-m^{2} \varphi
\end{gathered}
$$

The terms with angular derivatives in (2.6) are equal to

$$
-\frac{1}{R^{2}}\left[\partial_{\vartheta}^{2} \varphi+\cot \vartheta \partial_{\vartheta} \varphi+\frac{1}{\sin ^{2} \vartheta} \partial_{\phi}^{2} \varphi\right]=\frac{\vec{L}^{2} \varphi}{R^{2}}
$$

where $\vec{L}^{2}$ is the square of the angular momentum operator. Consequently the angular variables can be separated by means of spherical harmonics $Y_{l}^{m}(\vartheta, \phi)$ which satisfy

$$
\left[\partial_{\vartheta}^{2}+\cot \vartheta \partial_{\vartheta}+\frac{1}{\sin ^{2} \vartheta} \partial_{\phi}^{2}\right] Y_{l}^{m}=-l(l+1) Y_{l}^{m}(\vartheta, \phi) .
$$

Then writing the scalar field in the form

$$
\varphi=f(t, r) Y_{l}^{m}(\vartheta, \phi)
$$

there remains the radial equation

$$
\frac{1}{X R^{2}} \partial_{t}\left(X R^{2} \partial_{t} f\right)-\frac{1}{X^{2}} \partial_{r}^{2} f+\frac{l(l+1)}{R^{2}} f=-m^{2} \varphi
$$

to be solved. Since the radial variable $r$ appears only in the derivative it can also be separated by means of the product form

$$
f(t, r)=f_{0}(t) f_{1}(r)
$$

which yields

$$
\frac{X}{R^{2} f_{0}} \partial_{t}\left(X R^{2} \partial_{t} f_{0}\right)+l(l+1) \frac{X^{2}}{R^{2}}+m^{2} X^{2}=\frac{\partial_{r}^{2} f_{1}}{f_{1}}=\lambda
$$


Here the left side depends only on $t$ and the right side only on $r$, so both sides are constant $=\lambda$. We first assume a positive constant $\lambda=\mu^{2}$. Then we have

$$
f_{1}(r)=e^{-\mu r}
$$

with positive $\mu$ for a bounded solution. For a negative $\lambda=-k^{2}$ we get oscillating solutions

$$
f_{1}(r)=\sin k r \quad \text { or } \quad \cos k r .
$$

The remaining nontrivial equation for $f_{0}(t)$ is

$$
\partial_{t}\left(X R^{2} \partial_{t} f_{0}\right)+l(l+1) X f_{0}+m^{2} \frac{R^{2}}{X} f_{0}=\lambda \frac{R^{2}}{X} f_{0} .
$$

The left-hand side is a self-adjoint second order differential operator [8]. With suitable boundary conditions, equation (2.13) can be considered as a self-adjoint eigenvalue problem with eigenvalue $\lambda$. The spectrum is real and consists of a negative continuous spectrum $\lambda=-k^{2}$ and possible discrete positive eigenvalues $\lambda=\mu^{2}$. It follows from the completeness and expansion theorems [8] that any $L^{2}$-function $F(t)$ can be represented as a wave-packet of eigen-solutions. To see this explicitly let us consider the functions $X(t)$ and $R(t)$ in (2.13) in more detail.

The $t$-dependence of $X(t)$ and $R(t)$ is most conveniently given in parametric form [1]

$$
\begin{gathered}
t=T_{L}(w-\sin w \cos w) \\
X(t)=|\cot w|=\dot{R} \\
R(t)=T_{L} \sin ^{2} w
\end{gathered}
$$

where $\pi / 2<w<\pi$ and $T_{L}$ is of the order of the Hubble time. We are particularly interested in the behavior of the solution near the Big Bang $w=\pi / 2$. Therefore we set

$$
w=\frac{\pi}{2}+\tau
$$

so that

$$
\begin{gathered}
X(t)=|\tan \tau|=\tau+O\left(\tau^{3}\right), \quad R(t)=T_{L}\left(1+O\left(\tau^{2}\right)\right) \\
\partial_{t}=\frac{1}{2 T_{L}} \partial_{\tau}
\end{gathered}
$$

and we study the behavior for $\tau \rightarrow 0$.

For small $\tau$ and positive $\lambda=\mu^{2}$ equation (2.13) is of the form

$$
\tau^{2} \partial_{\tau}^{2} f_{0}+\tau \partial_{\tau} f_{0}+4\left[l(l+1)+T_{L}^{2} m^{2}\right] \tau^{2} f_{0}=4 T_{L}^{2} \mu^{2} f_{0}
$$

The solution is given by Bessel functions. For later use we note the following differential equation ([4], equ.9.1.53)

$$
\tau^{2} \ddot{y}+(1-2 p) \tau \dot{y}+\left(\lambda^{2} q^{2} \tau^{2 q}+p^{2}-\nu^{2} q^{2}\right) y=0
$$

with the solutions

$$
y=\tau^{p} Z_{\nu}\left(\lambda \tau^{q}\right)
$$

where $Z_{\nu}$ are Bessel functions. It follows that the solution of (2.18) is of the form

$$
f_{0}=Z_{\nu}\left(2 \sqrt{l(l+1)+m^{2} T_{L}^{2}} \tau\right)
$$

with

$$
\nu=2 T_{L} \mu \text {. }
$$

Now we consider the second possibility of a negative constant $\lambda=-k^{2}$ in (2.10) which corresponds to the continuous spectrum. Then we get the oscillating solutions (2.12) which describe propagating wave modes. The corresponding time depending equation (2.18) leads to Bessel functions with purely 
imaginary indices. This case has nicely been treated by Matyshev and Fohtung [9]. The real solution can be written in the form

$$
f_{0}(\tau)=A(\tau) \cos \left(2 T_{L} k \log \tau\right)+B(\tau) \sin \left(2 T_{L} k \log \tau\right)
$$

where

$$
\begin{aligned}
& A(\tau)=a_{0}+a_{2} \tau^{2}+\ldots \\
& B(\tau)=b_{0}+b_{2} \tau^{2}+\ldots
\end{aligned}
$$

are converging power series. The most important property of of the solution (2.23) is the possibility to get any singular behavior $\propto t^{-n}$ by forming wave packets. To see this let us consider the integral

$$
\int_{0}^{\infty} d k h(k) \sin \left(k 2 T_{L} \log \tau\right)=\frac{1}{\tau^{n}} .
$$

With the new variable $\xi=2 T_{L} \log \tau$ we have

$$
\int_{0}^{\infty} h(k) \sin k \xi d k=\exp -\left(\frac{n \xi}{2 T_{L}}\right)
$$

By inverting the Fourier integral we find

$$
h(k)=\frac{2}{\pi} \int_{0}^{\infty} \sin k \xi e^{-n \xi / 2 T_{L}} d \xi=\frac{2}{\pi} \frac{k}{k^{2}+\left(n / 2 T_{L}\right)^{2}} .
$$

To understand which solution is the physical one we consider the energy-momentum tensor of the massless scalar field

$$
T_{\alpha \beta}=\partial_{\alpha} \varphi \partial_{\beta} \varphi-\frac{1}{2} g_{\alpha \beta}\left(\partial_{\nu} \varphi \partial^{\nu} \varphi-m^{2} \varphi^{2}\right) .
$$

We know from the first order perturbation theory of the nonstandard background [1] that the energy density $T_{00}$ must diverge as $\tau^{-1}$ for $\tau \rightarrow 0$. This implies that

$$
\varphi \propto \tau^{1 / 2}
$$

Now, in case of the discrete spectrum, $\nu$ in $(2.22)$ must be equal to $1 / 2$ because the Bessel function $J_{\nu}(\tau)$ goes as $\tau^{\nu}$ for $\tau \rightarrow 0$. Then we conclude from (2.22)

$$
\mu=\frac{1}{4 T_{L}} .
$$

That means the radial variation (2.11) of the scalar field is of the scale of the present universe at early times. This is in sharp contrast to standard cosmology where the early universe is small. It has the consequence that the pressure $T_{11}$ is completely negligible in agreement with first order perturbation theory. Such a solution could describe some matter distribution in the universe.

\section{Maxwell Equations in Nonstandard Background}

Let $F^{\mu \nu}$ be the antisymmetric electromagnetic field tensor then the Maxwell equations in the presence of gravity are

$$
\begin{gathered}
\nabla_{\mu} F^{\mu \nu}=0 \\
\nabla_{\alpha} F_{\beta \gamma}+\nabla_{\beta} F_{\gamma \alpha}+\nabla_{\gamma} F_{\alpha \beta}=0
\end{gathered}
$$

where $\nabla_{\mu}$ denotes the covariant derivatives with respect to the metric $g_{\mu \nu}(2.3)$. The first equation (3.1) is the inhomogeneous Maxwell equation, we have assumed zero sources because we are not interested in 
the generation of the radiation. The second equation (3.2) is the homogeneous equation. It is well known that due to the antisymmetry of $F$ the covariant derivatives can be replaced by partial derivatives as follows [3]

$$
\begin{gathered}
\partial_{\mu}\left(\sqrt{g} F^{\mu \nu}\right)=0 \\
\partial_{\alpha} F_{\beta \gamma}+\partial_{\beta} F_{\gamma \alpha}+\partial_{\gamma} F_{\alpha \beta}=0 .
\end{gathered}
$$

To solve these equations the $4 \times 4$ field tensor is expressed by 3 -vectors $\vec{E}$ and $\vec{B}$ which are the electric and magnetic field strength, respectively. In rectangular Cartesian coordinates $\tilde{x}^{\mu}$ this form is given by $[4]$

$$
\tilde{F}^{\mu \nu}=\left(\begin{array}{cccc}
0 & -E^{x} & -E^{y} & -E^{z} \\
E^{x} & 0 & -B^{z} & B^{y} \\
E^{y} & B^{z} & 0 & -B^{x} \\
E^{z} & -B^{y} & B^{x} & 0
\end{array}\right) .
$$

Since we are calculating in spherical polar coordinates $x^{\mu}=(t, r, \vartheta, \phi)$ throughout, this tensor must be transformed according to

$$
F^{\alpha \beta}=\tilde{F}^{\mu \nu} \frac{\partial x^{\alpha}}{\partial \tilde{x}^{\mu}} \frac{\partial x^{\beta}}{\partial \tilde{x}^{\nu}} .
$$

The transformation matrix herein is equal to

$$
\frac{\partial x^{\alpha}}{\partial \tilde{x}^{\mu}}=\left(\begin{array}{cccc}
1 & 0 & 0 & 0 \\
0 & \sin \vartheta \cos \phi & \sin \vartheta \sin \phi & \cos \vartheta \\
0 & \frac{\cos \vartheta \cos \phi}{r} & \frac{\cos \vartheta \sin \phi}{r} & -\frac{\sin \vartheta}{r} \\
0 & -\frac{\sin \phi}{r \sin \vartheta} & \frac{\cos \phi}{r \sin \vartheta} & 0
\end{array}\right) .
$$

Then introducing the standard polar components

$$
\begin{gathered}
E^{r}=E^{x} \sin \vartheta \cos \phi+E^{y} \sin \vartheta \sin \phi+E^{z} \cos \vartheta \\
E^{\vartheta}=\frac{1}{r}\left(E^{x} \cos \vartheta \cos \phi+E^{y} \cos \vartheta \sin \phi-E^{z^{3}} \sin \vartheta\right) \\
E^{\phi}=\frac{1}{r \sin \vartheta}\left(E^{y} \cos \phi-E^{x} \sin \phi\right)
\end{gathered}
$$

and similarly for $\vec{B}$ we obtain

$$
F^{\alpha \beta}=\left(\begin{array}{cccc}
0 & -E^{r} & -E^{\vartheta} & -E^{\phi} \\
E^{r} & 0 & -B^{\phi} \sin \vartheta & \frac{B^{\vartheta}}{\sin \vartheta} \\
E^{\vartheta} & B^{\phi} \sin \vartheta & 0 & -\frac{B^{r}}{r^{2} \sin \vartheta} \\
E^{\phi} & -\frac{B^{\vartheta}}{\sin \vartheta} & \frac{B^{r}}{r^{2} \sin \vartheta} & 0
\end{array}\right) .
$$

The field tensor (3.9) is not yet the best form for the nonstandard background. Since we want to use the usual 3-dimensional differential operators curl and div we introduce additional factors $r^{2}$ into (3.9). This causes a slight modification of the meaning of electric and magnetic field strength $\vec{E}$ and $\vec{B}$ only. We shall calculate with the following final form of $F$ :

$$
F^{\alpha \beta}=\left(\begin{array}{cccc}
0 & -r^{2} E^{r} & -r^{2} E^{\vartheta} & -r^{2} E^{\phi} \\
r^{2} E^{r} & 0 & -r^{2} B^{\phi} \sin \vartheta & r^{2} \frac{B^{\vartheta}}{\sin \vartheta} \\
r^{2} E^{\vartheta} & r^{2} B^{\phi} \sin \vartheta & 0 & -\frac{B^{r}}{\sin \vartheta} \\
r^{2} E^{\phi} & -\frac{r^{2} B^{\vartheta}}{\sin \vartheta} & \frac{B^{r}}{\sin \vartheta} & 0
\end{array}\right) .
$$

We first consider the inhomogeneous equations (3.3). The zeroth component $\nu=0$ gives

$$
\partial_{r}\left(X R^{2} \sin \vartheta r^{2} E^{r}\right)+r^{2} \partial_{\vartheta}\left(X R^{2} \sin \vartheta E^{\vartheta}\right)+r^{2} X R^{2} \sin \vartheta \partial_{\phi} E^{\phi}=0 .
$$


After dividing by $r^{2} X R^{2} \sin \vartheta$ we get

$$
\frac{1}{r^{2}} \partial_{r}\left(r^{2} E\right)+\partial_{\vartheta} E^{\vartheta}+\cot \vartheta E^{\vartheta}+\partial_{\phi} E^{\phi}=0 .
$$

According to equ.(A.23) in the Appendix this is just the initial condition

$$
\operatorname{div} \vec{E}=0 .
$$

In the same way for $\nu=1,2,3$ we obtain the $r, \vartheta$ and $\phi$ components of the 3 -vector equation

$$
\partial_{t}\left(X R^{2} \vec{E}\right)=\operatorname{curl}\left(X R^{2} \vec{B}\right) .
$$

Here the time dependent factors $X R^{2}$ do not drop out.

The homogeneous equations (3.4) are more complicated. We first list the field tensor with lower indices:

$$
\begin{gathered}
F_{01}=X^{2} r^{2} E^{r}, \quad F_{02}=R^{2} r^{2} E^{\vartheta}, \quad F_{03}=R^{2} r^{2} \sin ^{2} \vartheta E^{\phi} \\
F_{12}=-X^{2} R^{2} r^{2} \sin \vartheta B^{\phi}, \quad F_{23}=-R^{4} B^{r} \sin \vartheta \\
F_{13}=X^{2} R^{2} r^{2} \sin \vartheta B^{\vartheta} .
\end{gathered}
$$

Now we write down the equation

$$
\partial_{1} F_{23}+\partial_{2} F_{31}+\partial_{3} F_{12}=0
$$

without time derivative. After dividing by $r^{2} \sin \vartheta$ we arrive at

$$
\frac{1}{r^{2}} \partial_{r}\left(R^{4} B^{r}\right)+\frac{1}{\sin \vartheta} \partial_{\vartheta}\left(\sin \vartheta X^{2} R^{2} B^{\vartheta}\right)+\partial_{\phi}\left(X^{2} R^{2} B^{\phi}\right)=0 .
$$

This is the divergence $\operatorname{div} \vec{b}=0$ of a new 3 -vector with components

$$
b^{r}=\frac{R^{4}}{r^{2}} B^{r}, \quad b^{\vartheta}=X^{2} R^{2} B^{\vartheta}, \quad b^{\phi}=X^{2} R^{2} B^{\phi} .
$$

This vector also appears in the other three equations which are of the form

$$
\partial_{t} \vec{b}=-\operatorname{curl} \vec{e}
$$

where $\vec{e}$ is a new electric vector with components

$$
e^{r}=X^{2} r^{2} E^{r}, \quad e^{\vartheta}=R^{2} E^{\vartheta}, \quad e^{\phi}=R^{2} E^{\phi} .
$$

Equation (3.17) is a modified form of the induction law.

The problem now is to solve the coupled equations (3.13) and (3.17). In vacuum this can be done by separating the angular variables by means of vector spherical harmonics. The standard reference for vector spherical harmonics is [5]. Unfortunately these authors use a convention which does not agree with tensor calculus. For example their contravariant and covariant polar components are the same. We therefore had to re-derive all formulas for spherical components, the results are given in the appendix. As in the vacuum without gravity there exist two classes of solutions, the so-called electric and magnetic multipoles [6].

\section{Electric Multipole Solution}

As in the vacuum case without gravity [6] we try a solution of the form

$$
\begin{gathered}
\vec{B}=\frac{g(t, r)}{r} \vec{Y}_{l m}^{(0)}(\vartheta, \phi) \\
\vec{E}=f_{1}(t, r) \vec{Y}_{l m}^{(1)}(\vartheta, \phi)+f_{2}(t, r) \vec{Y}_{l m}^{(-1)}(\vartheta, \phi) .
\end{gathered}
$$


A glance to the formulas (A.10) of the appendix shows that the inhomogeneous equation (3.13) is satisfied if the following two radial differential equations

$$
\begin{gathered}
\frac{1}{X R^{2}} \partial_{t}\left(X R^{2} f_{1}\right)=\frac{i}{r} \partial_{r} g \\
\frac{1}{X R^{2}} \partial_{t}\left(X R^{2} f_{2}\right)=\frac{i}{r^{2}} \sqrt{l(l+1)} g
\end{gathered}
$$

are fulfilled. The physical solutions of Maxwell equations must be real. But we work with complex spherical harmonics in (4.1-2) which is the usual convention. This leads to some imaginary factors in the equations. At the end we can always take real and imaginary parts in (4.1-2) to obtain real physical solutions.

For the homogeneous equation (3.17) we must calculate all three components separately. The $r$ component is simple because $\vec{Y}^{(0)}$ has no $r$-component. Then there is no time derivative and we get $\operatorname{div} \vec{E}=0$. For the $\vartheta$-component we obtain

$$
\partial_{t}\left(X^{2} R^{2} \frac{g}{r} \vec{Y}^{(0) \vartheta}\right)=-\frac{1}{r^{2} \sin \vartheta}\left[\partial_{\phi}\left(X^{2} r^{2} E^{r}\right)-\partial_{r}\left(r^{2} \sin ^{2} \vartheta R^{2} E^{\phi}\right]\right.
$$

The derivative $\partial_{\phi}$ gives a factor $\mathrm{im}$ from the spherical harmonics. Then applying the formulas in the appendix, all terms have a factor $m$ which drops out.

Multiplying the resulting equation by $\sqrt{l(l+1)} r^{2} \sin \vartheta / Y_{l}^{m}(\vartheta, \phi)$ we get

$$
\partial_{t}\left(X^{2} R^{2} g\right)=i r^{2} X^{2} \sqrt{l(l+1)} f_{2}-i R^{2} \partial_{r}\left(r f_{1}\right) .
$$

The same equation follows from the $\phi$-component of equ.(3.17). This proves that the angular dependence can indeed be separated by means of vector spherical harmonics.

Now the three coupled first order differential equation (4.3), (4.4) and (4.6) for $f_{1}, f_{2}$ and $g(t, r)$ must be solved. By suitable multiplication we write the equations as follows

$$
\begin{gathered}
\frac{1}{X R^{2}} \partial_{t}\left(X R^{2} r f_{1}\right)=i \partial_{r} g \\
\frac{1}{X R^{2}} \partial_{t}\left(X R^{2} r^{2} f_{2}\right)=i \sqrt{l(l+1)} r g \\
\frac{1}{R^{2}} \partial_{t}\left(X R^{2} g\right)-i \frac{X^{2}}{R^{2}} \sqrt{l(l+1)} r^{2} f_{2}=-i \partial_{r}\left(r f_{1}\right) .
\end{gathered}
$$

Now we can separate the variables $t$ and $r$ by means of the product ansatz

$$
\begin{gathered}
r f_{1}=u_{0}(t) u_{1}(r), \quad r^{2} f_{2}=v_{0}(t) v_{1}(r) \\
g=w_{0}(t) w_{1}(r) .
\end{gathered}
$$

Then equ.(4.7) becomes

$$
\frac{1}{X R^{2}} \frac{\partial_{t}\left(X R^{2} u_{0}\right)}{w_{0}(t)}=i \frac{\partial_{r} w_{1}(r)}{u_{1}(r)}=\lambda_{1}
$$

Here the left side depends on $t$ only and the right side on $r$. Consequently both sides are constant, this constant of integration is $\lambda_{1}$. Equ.(4.8) is treated in the same way with the result

$$
\frac{1}{X R^{2}} \frac{\partial_{t}\left(X R^{2} v_{0}\right)}{w_{0}(t)}=i \sqrt{l(l+1)} \frac{w_{1}(r)}{v_{1}(r)}=\lambda_{2}
$$

with a second constant of integration.

The third equation (4.9) assumes the following form

$$
\frac{1}{R^{2}} \partial_{t}\left(X^{2} R^{2} w_{0}\right) w_{1}(r)-i \frac{X^{2}}{R^{2}} \sqrt{l(l+1)} v_{0}(t) v_{1}(r)=-i \partial_{r} u_{1}(r) u_{0}(t) .
$$


To separate the variables we substitute

$$
w_{1}(r)=-\frac{i \lambda_{2}}{\sqrt{l(l+1)}} v_{1}
$$

from (4.13) herein and get

$$
\frac{-i \lambda_{2}}{\sqrt{l(l+1)}} \frac{1}{R^{2} u_{0}} \partial_{t}\left(X^{2} R^{2} w_{0}\right)-i \frac{X^{2}}{R^{2}} \sqrt{l(l+1)} \frac{v_{0}}{u^{0}}=-i \frac{\partial_{r} u_{1}(r)}{v_{1}(r)}=-i \lambda_{3} .
$$

We first integrate the $r$-dependence. Combining (4.12), (4.14) and (4.15) we get a second order equation for $u_{1}$ :

$$
\partial_{r}^{2} u_{1}=\lambda u_{1}
$$

with

$$
\lambda=\frac{\lambda_{1} \lambda_{3}}{\lambda_{2}} \sqrt{l(l+1)}=-k^{2} .
$$

We only consider the negative continuous spectrum which gives real oscillating radiative solutions again

$$
u_{1}(r)=\sin k r \quad \text { or } \quad \cos k r .
$$

A solution from the discrete spectrum is unphysical in the Maxwell case because a log-range cosmic electric field does not exist.

The time dependence is more complicated. From (4.12) and (4.13) we conclude

$$
u_{0}=\frac{\lambda_{1}}{\lambda_{2}} v_{0}
$$

Using this in (4.15) we find

$$
\partial_{t}\left(X^{2} R^{2} w_{0}\right)=\left[\frac{\lambda_{3}}{\lambda_{2}} \sqrt{l(l+1)} R^{2}-l(l+1) \frac{X^{2}}{\lambda_{1}}\right] u_{0} .
$$

On the other hand by differentiating (4.12) we have

$$
\lambda_{1} \partial_{t}\left(X^{2} R^{2} w_{0}\right)=\partial_{t}\left(X \partial_{t}\left(X R^{2} u_{0}\right)\right) .
$$

This allows to eliminate $w_{0}$ in $(4.20)$ and gives the following second order equation

$$
\left(X \partial_{t}\right)^{2} \tilde{u}_{0}+\frac{l(l+1)}{R^{2}} X^{2} \tilde{u}_{0}=-k^{2} \tilde{u}_{0}
$$

where we have introduced

$$
\tilde{u}_{0}=X R^{2} u_{0}
$$

and $k^{2}$ is given by $(4.17)$.

The equation (4.22) can be transformed into a 1-dimensional Schrödinger equation by introducing the variable

$$
\varrho(t)=\int^{t} \frac{d t^{\prime}}{X\left(t^{\prime}\right)}
$$

Then we have

$$
X \partial_{t}=X \frac{d \varrho}{d t} \frac{d}{d \varrho}=\frac{d}{d \varrho}
$$

so that

$$
\frac{d^{2} \tilde{u}_{0}}{d \varrho^{2}}+\frac{l(l+1)}{R^{2}} X^{2} \tilde{u}_{0}=-k^{2} \tilde{u}_{0}
$$

For $l=0$ we obtain the exact solution

$$
\tilde{u}_{0}=\sin k \varrho \quad \text { or } \quad \cos k \varrho .
$$


As we show in the last section this is also a good approximation for $l>0$ if $k$ is big. We also shall see that the frequency in (4.27) gets redshifted due to (4.24).

Finally, the magnetic function $g$ can be calculated from (4.12)

$$
\begin{gathered}
w_{0}(t)=\frac{1}{\lambda_{1} X R^{2}} \partial_{t}\left(X R^{2} u_{0}\right) \\
w_{1}(r)=-i \lambda_{1} \int u_{1}(r) d r=i \lambda_{1} \frac{\cos k r}{k},
\end{gathered}
$$

so that

$$
g=w_{0}(t) w_{1}(r)=\frac{i}{X R^{2}} \partial_{t}\left(X R^{2} u_{0}\right) \frac{\cos k r}{k}
$$

where the integration constant $\lambda_{1}$ drops out. The second electric function $f_{2}$ follows from (4.19) and $(4.14)$

$$
r^{2} f_{2}=v_{0}(t) v_{1}(r)=-\sqrt{l(l+1)} u_{0}(t) \frac{\cos k r}{k} .
$$

Again the integration constants $\lambda_{1}, \lambda_{2}$ cancel.

\section{Magnetic Multipole Solutions}

In these solutions the role of $\vec{E}$ and $\vec{B}$ is interchanged. We start from the ansatz

$$
\begin{gathered}
\vec{E}=\frac{f(t, r)}{r} \vec{Y}_{l m}^{(0)}(\vartheta, \phi) \\
\vec{B}=-\frac{1}{r}\left(g_{1}(t, r) \vec{Y}_{l m}^{(1)}(\vartheta, \phi)+g_{2}(t, r) \vec{Y}_{l m}^{(-1)}(\vartheta, \phi)\right) .
\end{gathered}
$$

The minus sign gives a different parity of the solution. Using the formulas in the Appendix it is easy to see that the angular dependence can again be separated. From the inhomogeneous Maxwell equation we get the following differential equation

$$
\frac{1}{X R^{2}} \partial_{t}\left(X R^{2} f\right)=-i \partial_{r} g_{1}+\frac{i}{r} \sqrt{l(l+1)} g_{2}
$$

The $r$-component of the homogeneous equation gives

$$
\partial_{t}\left(R^{4} g_{2}\right)=i r R^{2} \sqrt{l(l+1)} f
$$

and the $\vartheta$ and $\phi$-components both yield the equation

$$
\partial_{0}\left(X^{2} R^{2} g_{1}\right)=\frac{i}{r} R^{2} \partial_{r}(r f) .
$$

Note that the relation $\operatorname{div} \vec{E}=0$ follows from (5.1) due to (A.7) in the appendix.

To separate the $t$ and $r$ dependence we write

$$
\begin{gathered}
r f=u_{2}(t) u_{3}(r) \\
g_{1}=v_{2}(t) v_{3}(r), \quad g_{2}=w_{2}(t) w_{3}(r) .
\end{gathered}
$$

Then from equ.(5.4) we obtain

$$
\frac{1}{R^{2} u_{2}} \partial_{t}\left(R^{4} w_{2}\right)=i \sqrt{l(l+1)} \frac{u_{3}}{w_{3}}=\lambda_{4}
$$

and equ.(5.5) gives

$$
\frac{1}{R^{2} u_{2}} \partial_{t}\left(X^{2} R^{2} v_{2}\right)=\frac{i}{r} \frac{\partial_{r} u_{3}}{v_{3}}=\lambda_{5}
$$


Equation (5.3) reads

$$
\frac{1}{X R^{2}} \partial_{t}\left(X R^{2} u_{2}\right) u_{3}(r)=-i r v_{2}(t) \partial_{r} v_{3}(r)+i \sqrt{l(l+1)} w_{2} w_{3}
$$

Here we substitute $u_{3}$ by $w_{3}$ using (5.8) and then we can separate the variables

$$
\frac{1}{v_{2}}\left(\frac{\lambda_{4}}{\sqrt{l(l+1)}} \frac{\partial_{t}\left(X R^{2} u_{2}\right)}{X R^{2}}+\sqrt{l(l+1)} w_{2}\right)=\frac{r}{w_{3}} \partial_{r} v_{3}=\lambda_{6} .
$$

Again we first solve the $r$-dependence which now is more complicated. Combining (5.8) and (5.11) we find

$$
\partial_{r} v_{3}=i \frac{\lambda_{6}}{\lambda_{4} r} \sqrt{l(l+1)} u_{3} .
$$

On the other hand differentiating (5.9) we obtain

$$
\partial_{r} v_{3}=\frac{i}{\lambda_{5}} \partial_{r}\left(\frac{1}{r} \partial_{r} u_{3}\right)=i \frac{\lambda_{6}}{\lambda_{4} r} \sqrt{l(l+1)} u_{3} .
$$

Then we arrive at the final second order equation

$$
\partial_{r}\left(\frac{1}{r} \partial_{r} u_{3}\right)=-k^{2} \frac{u_{3}}{r}
$$

where

$$
k^{2}=-\frac{\lambda_{5} \lambda_{6}}{\lambda_{4}} \sqrt{l(l+1)} .
$$

The solution is given by the Bessel function of the first kind

$$
u_{3}=r J_{1}(k r) \text {. }
$$

To calculate the $t$-dependence we proceed as in the electric multipole case. From (5.8) and (5.9) we obtain

$$
v_{2}=\frac{\lambda_{5}}{\lambda_{4}} \frac{R^{2}}{X^{2}} w_{2}
$$

This is substituted into (5.11) yielding

$$
\frac{\lambda_{4}}{\sqrt{l(l+1)}} \frac{\partial_{t}\left(X R^{2} u_{2}\right)}{X R^{2}}+\sqrt{l(l+1)} w_{2}=\frac{\lambda_{5} \lambda_{6}}{\lambda_{4}} \frac{R^{2}}{X^{2}} w_{2} .
$$

Here $u_{2}$ can be eliminated by means of (5.11). Then we end up with the following second order equation for

$$
\begin{gathered}
\tilde{w}_{2}=R^{4} w_{2} \\
\partial_{t}\left(X \partial_{t} \tilde{w}_{2}+\frac{l(l+1)}{R^{2}} X \tilde{w}_{2}=-\frac{k^{2}}{X} \tilde{w}_{2} .\right.
\end{gathered}
$$

This agrees with (4.22).

After solving (5.19) for $\tilde{w}_{2}$ all functions can be calculated. The final results are

$$
\begin{gathered}
r f=\frac{i}{\lambda_{4} R^{2}} \partial_{t} \tilde{w}_{2} r J_{1}(k r) \\
g_{1}=\frac{R^{2}}{\lambda_{4} X^{2}} w_{2} i k J_{0}(k r) \\
g_{2}=\frac{i}{\lambda_{4}} \sqrt{l(l+1)} w_{2}(t) r J_{1}(k r) .
\end{gathered}
$$

Here the constant of integration $\lambda_{4}$ does not drop out, however it can be absorbed by a renormalization of the magnetic solution. 


\section{Discussion}

First we discuss the simple solution (4.18), (4.27)

$$
r f_{1}(t, r)=\sin k r \sin k \varrho(t) .
$$

The wavelength $\lambda$ is conventionally defined by

$$
k=\frac{2 \pi}{\lambda}
$$

and it remains constant along the light path by (6.1). The time-dependent factor in (6.1) defines the frequency $\omega(t)$ according to

$$
\omega(t)=\frac{d}{d t}\left(k \varrho(t)=\frac{k}{X(t)}=\frac{2 \pi}{\lambda X(t)} .\right.
$$

This frequency changes with time. If we compare the frequencies at time of emission with time of observation we get the redshift $z$

$$
1+z=\frac{\omega_{\mathrm{em}}}{\omega_{\mathrm{obs}}}=\frac{X\left(t_{\mathrm{obs}}\right)}{X\left(t_{\mathrm{em}}\right)}
$$

in agreement with previous results [1]. The phase velocity of the radiation is equal to

$$
c=\frac{\omega \lambda}{2 \pi}=\frac{1}{X(t)},
$$

so it is changed by the cosmic gravitational field. The result (6.5) is also equal to $d r / d t$ on the radial null geodesic. This cosmic variation of the light speed is embarrassing many people, because the same result follows in standard FLRW cosmology.

The results (6.3-5) can also be written as

$$
\frac{\nu(t)}{c(t)}=\frac{1}{\lambda}=\text { const. }
$$

independent of $t$. This is important for understanding CMB properly. The black-body spectral energy density is given by Planck's radiation law

$$
u(\nu, T)=\frac{8 \pi}{c^{3}} \nu^{2} \frac{h \nu}{\exp \left(h \nu / k_{B} T\right)-1}
$$

where the factor in front is the density of photon states

$$
d N=\frac{8 \pi}{c^{3}} \nu^{2} d \nu
$$

and the fraction is the corresponding mean energy of the states at temperature $T$. By (6.4) we have

$$
\nu_{\mathrm{em}}=(1+z) \nu_{\mathrm{obs}}
$$

which in the exponential yieds

$$
\frac{\nu_{\mathrm{em}}}{T_{\mathrm{em}}}=\frac{(1+z) \nu_{\mathrm{obs}}}{T_{\mathrm{em}}} \equiv \frac{\nu_{\mathrm{obs}}}{T_{\mathrm{obs}}}
$$

so that

$$
T_{\mathrm{obs}}=\frac{T_{\mathrm{em}}}{1+z} .
$$

According to (6.6) there is no further change of the $\nu^{3}$ factor in Planck's law (6.7). The reason is that the density of states (6.8) does not change, because the 3 -dimensional wave vectors $\vec{k}$ do not change as in (6.2). Only the energies $h \nu$ get redshifted. If one puts the light speed $c=1$ one misses the point. A 
factor $c_{0}$ in the metric (2.1) would change the unit of time, but this factor then appears in (6.5) and all conclusions remain valid.

In the magnetic multipole radiation the $r$-dependence (5.16) is now given by

$$
u_{3}=r J_{1}(k r)=r \sqrt{\frac{2}{\pi k r}} \cos (k r-3 \pi / 4)\left(1+O(k r)^{-1}\right) .
$$

Here we have $k r \gg 1$ because the wavelength of CMB is very small compared with cosmic distances. Therefore we can use the asymptotic expression for the Bessel function [7].

Now let us discuss higher multipoles $l>0$, in fact the lowest multipole is dipole radiation $l=1$. Until now the explicit time-dependence of $X(t), R(t)$ was not used, so that the separation of Maxwell equations by means of vector spherical harmonics is generally true. But now we restrict to the nonstandard background. We write (4.26) as a quantum mechanical Schrödinger equation

$$
\frac{d^{2} u}{d \varrho^{2}}+\left(k^{2}-V\right) u=0
$$

where

$$
V=-l(l+1) \frac{X^{2}}{R^{2}}=-l(l+1) \frac{T_{L}-R}{R^{3}} .
$$

To get the potential $V$ as a function of $\varrho(4.24)$ we use the parametric representation (2.14-15)

$$
\begin{gathered}
R(w)=T_{L} \sin ^{2} w \\
X(w)=-\cot w \\
\frac{d t}{d w}=2 T_{L} \sin ^{2} w .
\end{gathered}
$$

This gives

$$
\begin{gathered}
\varrho=\int^{t} \frac{d t}{-\cot w}=2 T_{L} \int^{w} \frac{\sin ^{2} w^{\prime}}{-\cot w^{\prime}} d w^{\prime}= \\
=T_{L}\left(2 \log \cos w+\sin ^{2} w\right) .
\end{gathered}
$$

Substituting $w$ by $R(6.9)$ we arrive at

$$
\varrho=R+T_{L} \log \left(1-\frac{R}{T_{L}}\right)
$$

This together with (6.14) yields the potential $V(\varrho)$. The Big Bang corresponds to $R=T_{L}$, that is $\varrho=-\infty$, where the potential vanishes. For $R \rightarrow 0$ we have $\varrho \rightarrow 0$ and a strong singularity $\approx \varrho^{-3}$ in the potential (6.14). This is the end of the Universe at $t=\pi T_{L}[1]$.

For cosmology it is sufficient to solve equ.(6.13) in the short-wave (WKB) approximation, that means for big $k$. To do so we assume the solution of the form

$$
u=\exp \left(i k \int^{\varrho} s\left(\varrho^{\prime}\right) d \varrho^{\prime}\right)
$$

Then (6.13) goes over into the following first order Riccati equation for $s(\varrho)$

$$
i k s^{\prime}+{ }^{2}\left(1-s^{2}\right)-V=0 .
$$

Here we substitute the power series

$$
s=\sum_{n=0}^{\infty} \frac{s_{n}(\varrho)}{k^{n}}
$$


and compare the powers of $k$. From $O\left(k^{2}\right)$ we get $s_{0}= \pm 1, O(k)$ gives $s_{1}=0$ and $O\left(k^{0}\right)$ yields

$$
s_{2}=-\frac{V}{2 s_{0}} .
$$

With $s_{0}=1$ we finally obtain

$$
\int s=\varrho-\frac{1}{2 k^{2}} \int^{\varrho} V\left(\varrho^{\prime}\right) d \varrho^{\prime} .
$$

The leading contribution $\varrho$ is our simple result (6.1). The next order gives a correction to the redshift which, however, is small $O\left(k^{-2}\right)$.

\section{Appendix}

Let $\vec{e}_{x}, \vec{e}_{y}, \vec{e}_{z}$ be Cartesian unit vectors and

$$
\vec{e}_{0}=\vec{e}_{z}, \quad \vec{e}_{1}=-\frac{\vec{e}_{x}+i \vec{e}_{y}}{\sqrt{2}}, \quad \vec{e}_{-1}=\frac{\vec{e}_{x}-i \vec{e}_{y}}{\sqrt{2}}
$$

The vector spherical harmonics are defined by

$$
\begin{aligned}
& \vec{Y}_{J M}^{L}(\vartheta, \phi)=\sum_{m, q} Y_{L}^{m}(\vartheta, \phi) \vec{e}_{q}(L m 1 q \mid L 1 J M) \\
& =(-)^{L+1+M} \sqrt{2 J+1} \sum_{m, q} Y_{L}^{m} \vec{e}_{q}\left(\begin{array}{ccc}
L & 1 & J \\
m & q & -M
\end{array}\right)
\end{aligned}
$$

where the Clebsch-Gordon coefficients or Wigner's $3 j$-symbols for spin 1 appear. For electromagnetic radiation the following linear combinations are most useful:

$$
\begin{gathered}
\vec{Y}_{J M}^{(0)}=\vec{Y}_{J M}^{J} \\
\vec{Y}_{J M}^{(1)}=\sqrt{\frac{J+1}{2 J+1}} \vec{Y}_{J M}^{J-1}+\sqrt{\frac{J}{2 J+1}} \vec{Y}_{J M}^{J+1} \\
\vec{Y}_{J M}^{(-1)}=\sqrt{\frac{J}{2 J+1}} \vec{Y}_{J M}^{J-1}-\sqrt{\frac{J+1}{2 J+1}} \vec{Y}_{J M}^{J+1} .
\end{gathered}
$$

$\vec{Y}(-1)$ is parallel to the radial unit vector $\vec{n}=\vec{x} / r$, the other two are perpendicular to $\vec{n}$.

The 3-dimensional differential operators div and curl operate as follows

$$
\begin{gathered}
\operatorname{div}\left[f(r) \vec{Y}_{J M}^{(1)}(\vartheta, \phi)\right]=-\sqrt{J(J+1)} \frac{f(r)}{r} Y_{J}^{M}(\vartheta, \phi) \\
\operatorname{div}\left[f(r) \vec{Y}_{J M}^{(0)}(\vartheta, \phi)\right]=0 \\
\operatorname{div}\left[f(r) \vec{Y}_{J M}^{(-1)}(\vartheta, \phi)\right]=\left(\partial_{r}+\frac{2}{r}\right) f(r) Y_{J}^{M}(\vartheta, \phi)
\end{gathered}
$$

and

$$
\begin{gathered}
\operatorname{curl}\left[f(r) \vec{Y}_{J M}^{(1)}(\vartheta, \phi)\right]=i\left(\partial_{r}+\frac{1}{r}\right) f(r) \vec{Y}_{J M}^{(0)}(\vartheta, \phi) \\
\operatorname{curl}\left[f(r) \vec{Y}_{J M}^{(0)}(\vartheta, \phi)\right]=i\left(\partial_{r}+\frac{1}{r}\right) f(r) \vec{Y}_{J M}^{(1)}(\vartheta, \phi)+i \sqrt{J(J+1)} \frac{f}{r} \vec{Y}_{J M}^{(-1)} \\
\operatorname{curl}\left[f(r) \vec{Y}_{J M}^{(-1)}(\vartheta, \phi)\right]=-i \sqrt{J(J+1)} \frac{f}{r} \vec{Y}_{J M}^{(0)}
\end{gathered}
$$

We do not use the nabla notation $\nabla$ because it is reserved for the 4-dimensional covariant derivatives. 
So far all formulas agree with ref.[5], the differences now appear when we use spherical polar coordinates. The transformation of coordinates must be carried out according to tensor calculus as described in sect.3. Then covariant and contravariant polar components are different. So we have the following contravariant components

$$
\begin{gathered}
(\operatorname{grad} f)^{r}=\partial_{r} f, \quad(\operatorname{grad} f)^{\vartheta}=\frac{1}{r^{2}} \partial_{\vartheta} f \\
(\operatorname{grad} f)^{\phi}=\frac{1}{r^{2} \sin ^{2} \vartheta} \partial_{\phi} f
\end{gathered}
$$

and

We also note

$$
\begin{gathered}
(\operatorname{curl} \vec{A})^{r}=\frac{1}{\sin \vartheta}\left[\partial_{\vartheta}\left(\sin ^{2} \vartheta A^{\phi}\right)-\partial_{\phi} A^{\vartheta}\right] \\
(\operatorname{curl} \vec{A})^{\vartheta}=\frac{1}{r^{2} \sin \vartheta}\left[\partial_{\phi} A^{r}-\sin ^{2} \vartheta \partial_{r}\left(r^{2} A^{\phi}\right)\right] \\
(\operatorname{curl} \vec{A})^{\phi}=\frac{1}{r^{2} \sin \vartheta}\left[\partial_{r}\left(r^{2} A^{\vartheta}\right)-\partial_{\vartheta} A^{r}\right] .
\end{gathered}
$$

$$
\operatorname{div} \vec{A}=\frac{1}{r^{2}} \partial_{r}\left(r^{2} A^{r}\right)+\frac{1}{\sin \vartheta} \partial_{\vartheta}\left(\sin \vartheta A^{\vartheta}\right)+\partial_{\phi} A^{\phi} .
$$

To apply these formulas we need the polar components of the vector spherical harmonics:

$$
\begin{gathered}
\vec{Y}_{J M}^{(1) r}=0, \quad \vec{Y}_{J M}^{(1) \vartheta}=\frac{\partial_{\vartheta} Y_{J}^{M}}{r \sqrt{J(J+1)}} \\
\vec{Y}_{J M}^{(1) \phi}=\frac{i M Y_{J}^{M}}{r \sin ^{2} \vartheta \sqrt{J(J+1)}} \\
\vec{Y}_{J M}^{(0) r}=0, \quad \vec{Y}_{J M}^{(0) \vartheta}=-\frac{M Y_{J}^{M}}{r \sin \vartheta \sqrt{J(J+1)}} \\
\vec{Y}_{J M}^{(0) \phi}=\frac{-i \partial_{\vartheta} Y_{J}^{M}}{r \sin \vartheta \sqrt{J(J+1)}} \\
\vec{Y}_{J M}^{(-1) r}=Y_{J}^{M}(\vartheta, \phi), \quad \vec{Y}_{J M}^{(-1) \vartheta}=0=\vec{Y}_{J M}^{(-1) \phi} .
\end{gathered}
$$

For completeness we list the polar components of the 3-dimensional vector product:

$$
\begin{gathered}
(\vec{a} \times \vec{b})^{r}=r^{2} \sin \vartheta\left(a^{\vartheta} b^{\phi}-a^{\phi} b^{\vartheta}\right) \\
(\vec{a} \times \vec{b})^{\vartheta}=\sin \vartheta\left(a^{\phi} b^{r}-a^{r} b^{\phi}\right) \\
(\vec{a} \times \vec{b})^{\phi}=\frac{1}{\sin \vartheta}\left(a^{r} b^{\vartheta}-a^{\vartheta} b^{r}\right) .
\end{gathered}
$$

\section{References}

1. Scharf G., 2019, Perturbation theory in nonstandard cosmology, Advances Astrophys.4 (4), 133 arXiv 1905.02526

2. Scharf G., 2020, Cosmology without dark matter, Advances Astrophys. 5 (1), 1

3. Weinberg St.,1972, Gravitation and Cosmology, John Wiley, Sons

4. Scharf G., 1994, From Electrostatics to Optics, Springer-Verlag, Berlin

5. Varshalovich D.A., Moskalev A.N. Khersonskii V.K.,1988, Quantum Theory of Angular Momentum, World Scientific

6. Blatt J.M.,Weisskopf V.F.,1952, Theoretical Nuclear Physics, John Wiley, Sons

7. Abramowitz M., Stegun I.A., 1965, Handbook of Mathematical Functions, Dover Publ. Inc., New York

8. Coddington E.A., Levinson N.,1955, Ordinary differential equations, McGraw-Hill Book Comp., Inc., New York

9. MatyshevA.A., Fohtung E.,2009, On the Computation and Application of Bessel Functions with Pure Imaginary Indices, arXiv:0910.0365 